\title{
Inhibitory Effects of Probenecid on Pharmacokinetics of Tenofovir Disoproxil Fumarate and Emtricitabine for On-Demand HIV Pre-Exposure Prophylaxis
}

Stephanie N. Liu, PharmD1, Brandon T. Gufford, PharmD, PhD1, Jessica Bo Li Lu1, Lane R. Bushman², Peter L. Anderson², PharmD, Richard F. Bergstrom, PhD¹, Zeruesenay Desta, PhD', Samir K. Gupta, MD, MS 3

${ }^{1}$ Division of Clinical Pharmacology, Department of Medicine, School of Medicine, Indiana University, Indianapolis, IN

2Department of Pharmaceutical Sciences, Skaggs School of Pharmacy and Pharmaceutical Sciences, University of Colorado, Aurora, CO

${ }^{3}$ Division of Infectious Diseases, Department of Medicine, School of Medicine, Indiana University, Indianapolis, IN

Corresponding Author: Samir K. Gupta, MD, MS; Emerson Hall Suite 421, 545 Barnhill Drive, Indianapolis, IN 46202, USA; email: sgupta1@iu.edu; phone: 317-274-7926; fax: 317-274-1587

Conflict of Interest Statement:

SKG is the site Principal Investigator for ongoing studies supported by Gilead Sciences, Inc. and GlaxoSmithKline/ViiV. He has also received advisory fees and travel support from Gilead Sciences, Inc. and GlaxoSmithKline/ViiV. He also has an investigator-initiated grant proposal under review with GlaxoSmithKline/ViiV.

PLA receives contract and research support from Gilead Sciences and paid to his institution.

All other authors declared no competing interests for this work.

Funding Information:

This work was supported by the Campbell Foundation for AIDS Research. In addition, this project was supported, in part, from the Indiana Clinical and Translational Sciences Institute funded, in part by Award Number UL1TR002529 from the National Institutes of Health, National Center for Advancing Translational Sciences, Clinical and Translational Sciences Award. The content is solely the responsibility of the authors and does not necessarily represent the official views of the National

This is the author's manuscript of the article published in final edited form as:

Liu, S. N., Gufford, B. T., Lu, J. B. L., Bushman, L. R., Anderson, P. L., Bergstrom, R. F., ... Gupta, S. K. (2019). Inhibitory Effects of Probenecid on Pharmacokinetics of Tenofovir Disoproxil Fumarate and Emtricitabine for On-Demand HIV Pre-

Exposure Prophylaxis. Clinical Pharmacology \& Therapeutics, n/a(n/a). https://doi.org/10.1002/cpt.1714 
Institutes of Health. SNL and BTG were supported by the National Institute of General Medical Sciences [Grant T32 GM008425]. BTG was also supported by the Indiana Clinical and Translational Sciences Institute Young Investigator Award [Grant UL1 TR001108]. ZD was also supported by the National Institute of General Medical Sciences [Grants R01s GM078501 and GM121707].

Abbreviations: PrEP, pre-exposure prophylaxis; HIV, human immunodeficiency virus; $\mathrm{CrCl}$, creatinine clearance; STIs, sexually transmitted infections; TDF, tenofovir disoproxil fumarate; TFV, tenofovir; FTC, emtricitabine; TFV-DP, tenofovir diphosphate; FTC-TP, emtricitabine triphosphate; PBMCs, peripheral blood mononuclear cells; LC-MS/MS, liquid chromatography-tandem mass spectrometry; $\mathrm{AE}$, adverse event; PRO, probenecid

Keywords: HIV, Pre-exposure prophylaxis, probenecid, tenofovir, emtricitabine

\section{Abstract}

In a randomized, cross-over pharmacokinetic study in healthy volunteers $(N=14)$, a single dose of $2 \mathrm{~g}$ probenecid (PRO)-boosted $600 \mathrm{mg}$ tenofovir disoproxil fumarate (TDF) / $400 \mathrm{mg}$ emtricitabine (FTC) (Treatment, T +PRO) was compared with the current On-Demand HIV PreExposure Prophylaxis (PrEP) from the IPERGAY study (a $600 \mathrm{mg}$ TDF/400 mg FTC on day 1 and $300 \mathrm{mg}$ TDF/200 mg FTC on days 2 and 3) (Control, C IPERGAY). PRO increased mean single dose plasma $\mathrm{AUC}_{0-\infty, \mathrm{SD}}$ of TFV and FTC by $61 \%$ and $68 \%$, respectively. The TFV-diphosphate (TFVDP) concentrations in peripheral blood mononuclear cells (PBMC) were higher ( 30\%) at 24 hours in T +PRO but then fell significantly lower $(\sim 40 \%)$ at 72 hours compared to C IPERGAY. The interaction between FTC and PRO was unexpected and novel. Further study is needed to determine if this PRO-boosted TDF/FTC regimen would be clinically effective.

This article is protected by copyright. All rights reserved 


\section{Introduction}

The prevention of human immunodeficiency virus (HIV) transmission is a worldwide imperative. Men who have sex with men (MSM) and transgender women (TGW) are 24 and 49 times more likely to acquire HIV than the general population, respectively (1). The mainstay for preexposure prophylaxis (PrEP) in HIV-negative individuals is the use of a combination pill containing $300 \mathrm{mg}$ of tenofovir disoproxil fumarate (TDF) and $200 \mathrm{mg}$ of emtricitabine (FTC), originally marketed as Truvada® (2). While many new drugs or formulations are under development for HIV PrEP, Truvada® is one of two agents currently FDA-approved for HIV PrEP (the other being Descovy®, or tenofovir alafenamide with FTC). It has currently four approved therapeutic equivalents or generics that may increase the accessibility and affordability of TDF/FTC for HIV PrEP worldwide. The availability and use of TDF/FTC for HIV PrEP therapy is linked to a significant reduction in new HIV infections in the US (3).

Traditionally, once daily oral dosing of $300 \mathrm{mg}$ TDF/200 mg FTC demonstrated a 92\% reduction of new HIV infections in MSM and TGW at high risk for infection when coupled with detectable drug levels reflecting compliance (4). The IPERGAY study, an "On-Demand" dosing regimen of 2 tablets of $300 \mathrm{mg}$ TDF/200 mg FTC 2-24 hours prior to HIV exposure and 1 tablet of $300 \mathrm{mg}$ TDF/200 mg FTC every 24 hours after sexual activity for 2 doses resulted in an $86 \%$ reduction in new HIV infections in MSM and TGW (5). Thus, On-Demand dosing showed comparable reduction in HIV transmissions to traditional daily HIV PrEP with the benefit of significantly less drug dosing requirements. The promise of effective HIV prophylaxis with traditional or On-Demand HIV PrEP regimens is highly dependent on the population and adherence (6).

Medication adherence is a major concern in individuals at high risk for acquiring HIV and crucial for effective protection from HIV acquisition $(4,6)$. Daily or fixed-duration dosing regimens can be burdensome, complex, and expensive for patients. As a result, a single oral dose of TDF/FTC for HIV PrEP could be more advantageous for patient adherence, particularly as an On-Demand HIV PrEP.

Our evaluation of the pharmacokinetic interaction with probenecid (PRO) seeks to evaluate a "boosting strategy" for TDF/FTC using PRO as a booster for the single-dose TDF/FTC kinetics for On-Demand HIV PrEP. Historically, PRO was used to minimize renal secretion of other anti-infective agents via organic anion transporters (OAT1 or OAT3) inhibition (7). Inhibition of renal elimination with PRO increases or prolongs exposure of several anti-infectives (i.e. penicillin, cefazolin) (7). Tenofovir (TFV), the TDF moiety found in circulation, exhibits similarly active renal secretion

This article is protected by copyright. All rights reserved 
mediated via OAT1 and OAT3 transporters, making TFV an attractive target for the inhibitory effect of PRO (8). The PRO "boosting" TDF drug-drug interaction (DDI) may provide clinical benefit, simplified dosing and improved adherence, and offer a cost-effective and accessible single-dose alternative for On-Demand HIV PrEP as per the IPERGAY regimen. These potential advantages could improve widespread uptake of PrEP in both resource-replete and resource-limited settings. Thus, we studied the pharmacokinetics of TDF and FTC in combination with PRO and compared it to the current 3-day TDF/FTC IPERGAY HIV PrEP regimen. A prospective, randomized, open-label, two-phase, active-control, pivotal, cross-over, pharmacokinetic study was conducted in healthy volunteers to compare the plasma, urine, and peripheral blood mononuclear cells (PBMC) pharmacokinetics of single-dose PRO with TDF/FTC to the current 3-day IPERGAY HIV PrEP regimen.

\section{Methods}

\section{Study population}

Inclusion. Non-smoker male participants (18-55 years old) within $32 \%$ of their ideal body weight and determined to be healthy through pre-enrollment medical assessment (medical history, laboratory blood and urine tests) were enrolled. The study was limited to males to minimize intersubject variance due to known sex-based differences in TFV disposition (9). Eligible subjects agreed to refrain from taking medications and herbal/dietary supplements that may interact with TDF, FTC, or PRO for at least two weeks prior to the start of the study and through study completion. The protocol was approved, in advance, by the Indiana University School of Medicine Institutional Review Board (IRB). All volunteers provided written informed consent that was approved by the Indiana University IRB. The study was performed in accordance with ethical standards in the Declaration of Helsinki. The study is registered in ClinicalTrials.gov under NCT03202511.

Exclusion. Subjects were excluded if they were female or had insufficient renal function $(\mathrm{CrCl} \leq 95 \mathrm{~mL} / \mathrm{min})$, current major or chronic illness (i.e. hypertension, HIV antibody positive, 
hepatitis B surface antigen positive), blood donations within the past two months, or conditions that placed the subject at a higher risk for contracting HIV during the study period (i.e. drug abuse, history of sexually transmitted infections, $>1$ sexual partner in the past 6 months).

\section{Study Design}

A prospective, randomized, open-label, two-treatment, active-control, pivotal, cross-over, pharmacokinetic study was conducted in healthy volunteers to compare the plasma, urine, and peripheral blood mononuclear cells (PBMC) pharmacokinetics of single-dose PRO with TDF/FTC to the current 3-day IPERGAY HIV PrEP regimen. Participants were randomized (SAS, PROC RANDOM, v. 9.4) by blocks of 4 to initially receive one of two sequences of treatments: (1) the test ("T") treatment (2g PRO and 600mg TDF / 400mg FTC on day 1 only) and (2) the control ("C") treatment IPERGAY (600mg TDF / 400mg FTC on day 1, 300mg TDF / 200mg FTC on days 2 and 3) (Figure 4). The wash out was a period of at least 6 weeks before participants were assigned to the other study treatment. All medications were purchased, stored, and dispensed by the Indiana University Investigational Drug Services (IDS) Pharmacy (TDF/FTC NDC\#:61958-0701-01; PRO NDC\#:00378-0156-01 / 68084-0945-25). All medications were administered by trained personnel in the Indiana University Clinical Research Center (CRC).

Procedures and Visits. All study procedures and visits were conducted at the CRC and identical for both test and control treatments with the exception of drug administration regimens. Participants arrived at the CRC for drug administration, blood and urine collection, and safety evaluation on days 1-4 of the study. The first dose of drug was administered on Day 1 at time (t) $0 \mathrm{~h}$ after an overnight fast. Subjects were served a full meal 4 hours after dosing on Day 1. All doses were administered with $\sim 250 \mathrm{~mL}$ water. Participants stayed overnight in the CRC days 1 through 2 (0-24 hours), followed by $\sim 1$ hour outpatient visits on days 3 (48 hour) and 4 (72 hour). On day 4, subjects returned their urine jugs and the final blood draw was collected before completion of the treatment period.

Sample Collections. Samples collected included (1) blood (plasma, isolated PBMCs) and (2) urine. Blood samples were drawn $(\sim 10 \mathrm{~mL})$ at $0,0.5,1,2,4,8,12,18,24,48$, and 72 hours postdose. Plasma was harvested for TFV and FTC bioanalytical and pharmacokinetic analysis. Peripheral blood mononuclear cell (PBMC) samples were obtained at $0,24,48$, and 72 hours. PBMC samples were used for TFV-DP \& FTC-TP bioanalytical and pharmacokinetic analysis. Blood samples for the first 24 hours were collected through a sterile indwelling catheter and by peripheral needle sticks for the 48- and 72-hours samples. A urine sample was collected at baseline (spot

This article is protected by copyright. All rights reserved 
collection) and all voided urine was collected for the entire 72 hours following dosing. Voided urine was collected for 12-hours intervals for the first 24 hours followed by 24-hours intervals for 24-48 and 48-72 hours. Urine from each time interval was collected in a different container. After the first 24 hours, urine void was collected at home and subjects were instructed to return urine containers at the following visit. Two 10-ml urine aliquots were saved from each time interval after the total urine volume is recorded. All blood samples were processed immediately following collection and stored by the Indiana University Clinical and Translational Science Laboratory (CTSL). Plasma was separated by centrifugation at $1200 \mathrm{~g}$ for $10 \mathrm{~min}$. PBMCs were first isolated using ficol gradient utilizing lymphocyte separation medium (LSM), phosphate-buffered saline (PBS) solution, centrifugation at $1800 \mathrm{~g}$ for $20 \mathrm{~min}$ and collection of the buffy coat layer containing the lymphocytes for counting and analysis (9). The PBMCs were then freshly lysed in cold $70 \%$ methanol to stabilize nucleotides during long term storage and then ambient during the analytical extraction process. Plasma, urine and PBMC samples were immediately stored at $-80^{\circ} \mathrm{C}$ pending analysis.

Safety Evaluation. Subjects were asked to report adverse events (AEs) to study investigators and CRC personnel. Participants who received at least one dose of TDF/FTC were included in the safety analysis. AEs were documented and reported to the Indiana University IRB. AEs were assessed by $\mathrm{NCl}$ Common Toxicity Criteria guidelines and internal safety reviews.

\section{Objectives and Endpoints}

Primary. The primary objective was to determine if the plasma and intracellular exposure of TFV/TFV-DP from a single PRO-boosted TDF regimen ("T" test treatment) were comparable to the current 3-day 'IPERGAY' unboosted TDF dosing regimen ("C" control treatment). The primary endpoints were pre-specified as the geometric mean ratio $(G M R)$ of plasma $\left(A \cup C_{0-72 h, p l a s m a}\right)$ and PBMC (AUC0 $-72 h, P B M C)$ exposure in the $T$ versus $C$ treatment arms.

Secondary. The secondary outcome was to compare the short-term safety and tolerability of the $T$ versus $C$ treatments.

\section{Quantification of drugs and active metabolites.}

Plasma. TFV and FTC were simultaneously quantified from plasma samples utilizing a validated liquid chromatography-tandem mass spectrometry (LC-MS/MS) assay previously published for TFV and FTC (10). Briefly, analytes chromatographic separation were done using a Synergi Polar-RP column $(100 \times 2.0 \mathrm{~mm}$ i.d.; $2.5 \mu \mathrm{m}$ particle size; Phenomenex, Torrance, CA $)$ using a mobile phase gradient consisting of acetonitrile containing $0.1 \%$ formic acid and water containing $0.1 \%$ formic acid, flow rate of $0.3 \mathrm{~mL} / \mathrm{min}$ and injection volume of $5 \mu \mathrm{L}$. Then, QTRAP $6500+\mathrm{MS}$ 
fitted with electrospray ionization was performed for analyte quantification. The total run time was 5 min. The lower limit of quantification (LLOQ) for TFV and FTC was 0.586 and $2.93 \mathrm{ng} / \mathrm{mL}$, respectively. The inter- and intra-day precision and accuracy for the assay was $\pm 10 \%$ and $\pm 2 \%$, respectively.

Peripheral Blood Mononuclear Cells. Tenofovir diphosphate (TFV-DP) and FTC triphosphate (FTC-TP) were quantified at the Colorado Antiviral Pharmacology Laboratory (deidentified) University of Colorado, using validated and routinely performed LC-MS/MS assays (11). The lower limit of quantification (LLOQ) for TFV-DP and FTC-TP was 5 to 10 or $100 \mathrm{fmol} / \mathrm{sample}$, respectively.

Urine. TFV and FTC were quantified simultaneously in urine by the LC-MS/MS assay method analogous to the method for plasma (10). Urine samples were diluted 100 -fold prior to analysis. The LLOQ for TFV and FTC were the same as mentioned above. The inter- and intra-day precision and accuracy for the assay was $\pm 18 \%$ and $\pm 2 \%$, respectively.

\section{Pharmacokinetic}

For the primary endpoint, area under the plasma concentration-time curve from zero to 72 hours $\left(\mathrm{AUC}_{0-72 \mathrm{~h} \text {,plasma }}\right)$ for the $\mathrm{T}$ and $\mathrm{C}$ arm were estimated using nonparametric superposition ( $\mathrm{C}$ arm only) and conventional non-compartmental analysis (NCA) ( $T$ and $\mathrm{C}$ arm) performed by Phoenix $®$ WinNonlin ${ }^{\circ}$ (version 6.4; Certara, Princeton, NJ). For the single-dose drug administration in the $\mathrm{T}$ $+\mathrm{PRO}$ treatment, $\mathrm{AUC}_{0-72 \mathrm{~h} \text {,plasma }}$ was calculated using the observed 0-72h plasma concentration data and estimated using NCA analysis only. For the multiple-dose drug administration in the C IPERGAY treatment, $A \cup C_{0-72 h \text {,plasma }}$ was estimated as the sum of the $A \cup C_{0-24 h}$ for the $1^{\text {st }}$ dose from the observed plasma concentration data using NCA in addition to the predicted $\mathrm{AUC}_{24-48 \mathrm{~h}}$ and $\mathrm{AUC}_{48-72 \mathrm{~h}}$ for the $2^{\text {nd }}$ and $3^{\text {rd }}$ doses obtained via nonparametric superposition and NCA (Figure 3). The predicted TFV and FTC plasma trough concentrations $(24,48,72 \mathrm{~h})$ from nonparametric superposition were compared to observed to assess for accuracy. The predicted concentrations were on average $\pm 15 \%$ of observed concentrations. The area under the PBMC concentration-time curve from zero to 72 hours $\left(A \cup C_{0-72 h, P B M C}\right)$ for the $T+P R O$ and $C$ IPERGAY treatments were calculated using the observed PBMC concentration data and estimated using NCA analysis ( $T$ and $C$ treatments) performed by Phoenix ${ }^{\circledR}$ WinNonlin ${ }^{\circledR}$. All AUC estimates were determined using the trapezoidal rule with linearup/log-down interpolation.

Single-dose DDI pharmacokinetic parameters were also explored and estimated using plasma and urine concentration data collected from 0-24 hours (incorporating only single dose of 272

This article is protected by copyright. All rights reserved 
mg TFV/400mg FTC) using conventional NCA performed by Phoenix® WinNonlin®. Pharmacokinetic outcomes included elimination rate constant $\left(\mathrm{k}_{\mathrm{el}}\right)$, maximum plasma concentration $\left(\mathrm{C}_{\max }\right)$, time of maximum plasma concentration $\left(t_{\max }\right)$, single-dose area under the plasma concentration-time curve from zero extrapolated to infinity $\left(\mathrm{AUC}_{0-\infty, \mathrm{SD}}\right)$, single-dose area under the plasma concentration curve from time zero to 24 hours $\left(\mathrm{AUC}_{0-24 \mathrm{~h}, \mathrm{SD}}\right)$, apparent volume of distribution $\left(\mathrm{V}_{\mathrm{z}} / \mathrm{F}\right)$, and apparent oral clearance (CL/F). Since TFV and FTC observe biphasic elimination kinetics in the dataset, the terminal half-life $\left(t_{1 / 2}\right)$ was calculated as $t_{1 / 2}=0.693 / k_{\text {el }}$ using the $0-24$ hours (alpha) and 0-72 hours from the $T+P R O$ treatment only (beta). Urinary excretion was calculated using Phoenix ${ }^{\circledR}$ WinNonlin $®$. Urine concentrations were multiplied by total urine volume collected during the interval and summed, $A_{e 0-24 h}$ to calculate amount excreted in the urine $\left(A_{e}\right)$. The percentage of the dose in urine was calculated by: $\%$ dose in urine $=A_{e}(m g) / 272 m g$ for TFV or $A_{e}(m g) / 400 m g$ for FTC. Renal

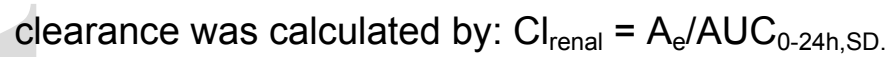

Pharmacokinetic outcomes are reported as geometric mean (GM), geometric mean ratio (GMR), and $90 \%$ confidence interval $(90 \% \mathrm{Cl})$. Data visualization (concentration-time profiles) is performed using GraphPad Prism v8.2.0 (San Diego, CA).

\section{Statistical}

Prior to performing the study, power and sample size were determined using intra-subject variability estimates from a previous study of daily TDF/FTC in healthy volunteers (12). To demonstrate bioequivalence, a total of 14 subjects with complete data were projected to provide $90 \%$ power to detect a mean difference of no more than $25 \%$ in the primary endpoint assuming a type I error rate of 0.05 and intra-subject variability as previously published (12). If the $90 \% \mathrm{Cl}$ of the $\mathrm{AUC}_{0 \text { - }}$ 72h,plasma and $\mathrm{AUC}_{0-72 \mathrm{~h}, \mathrm{PBMC}} \mathrm{GMR}$ were contained entirely within the pre-defined boundaries of 0.8 1.25 , then the probenecid "boosting" strategy would be considered pharmacokinetically bioequivalent to the IPERGAY regimen.

This article is protected by copyright. All rights reserved 


\section{Results}

\section{Participant Demographics}

The enrolled healthy male volunteers $(n=15)$ were self-identified Caucasian $(n=10)$, Asian $(n=2)$, Black $(n=2)$, and biracial $(n=1)$. The median age of participants was 26 years (range: 18-47 years) with a median height of 69 inches (range: 64-74) and weight of $75 \mathrm{~kg}$ (range: 59-86). All participants had a baseline $\mathrm{CrCl}>90 \mathrm{~mL} / \mathrm{min}$ with a median $\mathrm{SCr}$ of $1 \mathrm{mg} / \mathrm{dL}$ (range: $0.72-1.23 \mathrm{mg} / \mathrm{dL}$ ).

\section{Plasma and Urine Pharmacokinetics of TFV and FTC}

The plasma concentration-time profiles of the two dosing regimens ( $T+P R O(2 \mathrm{~g})$ treatment vs C IPERGAY treatment) in 14 healthy volunteers are shown in Figure 1. The concentration-time profiles up to 24 hours capture the overall kinetics of the loading dose in the presence and absence of PRO for both dosing regimens. The last two time points at 48 and 72 hours are to be interpreted differently for the two dosing regimens. For the T +PRO treatment, the 48 and 72 hour time points represent a continuation of the elimination phase (1-dose regimen). For the C IPERGAY treatment, the 48 and 72 hour time points represent the trough concentrations, $C_{t r}$, for the $2^{\text {nd }}$ and $3^{\text {rd }}$ doses (3dose regimen). The PK effects from a single dose of PRO given with single doses of TFV (272mg) and FTC (400 mg) was also estimated and derived using the sampling time of 24 hours (Table 1). When a single dose of TDF and FTC was co-administered with $2 \mathrm{~g} P R O$, overall exposure (AUC $\left.0_{0-\infty, S D}\right)$ of TFV and FTC increased by approximately $60-70 \%$ in the plasma. TFV and FTC apparent oral clearance, CL/F, significantly decreased by nearly $40 \%$ in the presence of PRO. A similar trend was observed for the apparent volume of distribution, $V_{z} / F$, of both TFV and FTC. Renal clearances of single dose TFV and FTC were significantly decreased in the presence of PRO. There was no difference in the estimated $t_{1 / 2}$ of TFV and FTC between treatments from the single dose data. However, the literature suggests TFV and FTC demonstrate biphasic elimination and are long halflife drugs ( $t_{1 / 2} \sim 18 \mathrm{~h}$ for TFV; $t_{1 / 2} \sim 10 \mathrm{~h}$ for FTC) (13). Thus, the terminal elimination $t_{1 / 2}$ of TFV and FTC could not be accurately estimated from the short sampling time of 0-24 $\mathrm{h}$ and suggests the single-dose data up to 24 hours appears to primarily capture the distribution phase only of TFV and FTC. Since the T +PRO treatment is comprised of a single dose regimen, the concentration-time data over 72 hours in the T +PRO treatment was used to estimate the true terminal $t_{1 / 2}$ with the DDI (+PRO). Since the C IPERGAY treatment has additional dosing at 24 and 48 hours, the true terminal $t_{1 / 2}$ could not be measured from our data. Instead the terminal $t_{1 / 2}$ from the $T+P R O$ treatment (Table 1) was compared to the literature and corresponds well ( $t_{1 / 2} \sim 18 \mathrm{~h}$ for TFV; $t_{1 / 2} \sim 10 \mathrm{~h}$ for FTC) (13) suggesting PRO does not have an effect on the terminal $t_{1 / 2}$ of TFV or FTC. PRO did not significantly

This article is protected by copyright. All rights reserved 
change maximum plasma concentration $\left(\mathrm{C}_{\max }\right)$ or time to reach maximum plasma concentration ( $T_{\max }$ ) of TFV. There was a $20 \%$ increase in FTC $C_{\max }$ for the $\mathrm{T}+\mathrm{PRO}$ treatment.

\section{Intracellular Exposure of TFV-DP and FTC-TP}

The intracellular concentration-time profiles and outcomes of TFV-DP and FTC-TP between the $\mathrm{T}+\mathrm{PRO}$ and $\mathrm{C}$ IPERGAY treatments are shown in Figure 2 and Table 2. At 24 hours, TFV-DP and FTC-TP concentrations in the T +PRO treatment were on average $30 \%$ and $10 \%$ higher than the C IPERGAY treatment, respectively. The two additional doses of TDF in the C IPERGAY treatment resulted in nonlinear accumulation of TFV-DP. In the C IPERGAY treatment, TFV-DP intracellular concentrations doubled after the second dose $\left(\mathrm{C}_{48 \mathrm{~h}}\right)$. FTC-TP concentrations did not appear to increase with the additional doses and demonstrated wide variability suggesting accumulation of FTC-TP is unlikely. In the T +PRO treatment, TFV-DP concentrations remained consistent over the 72 hours following single-dose administration, but FTC-TP concentrations steadily decreased over

72 hours. At both 48 hours and 72 hours, TFV-DP and FTC-TP concentrations were significantly higher in the C IPERGAY treatment compared to $T+P R O$ treatment.

\section{Bioequivalence Test for the Primary and Exploratory Endpoint}

The $A_{U} C_{0-72 h, p l a s m a} A U C_{0-72 h, P B M C}$ ratio of the $T+P R O$ treatment compared to C IPERGAY treatment for TFV and FTC are shown in Table 3. Since the C IPERGAY treatment was a 3-dose regimen and observed $\mathrm{PK}$ data well-characterized the loading dose only ( $1^{\text {st }}$ dose) along with trough concentrations for the $2^{\text {nd }}$ and $3^{\text {rd }}$ dose, the plasma $A U C_{0-72 h}$ in the $C$ IPERGAY treatment was estimated using a combination of both nonparametric superposition for the $2^{\text {nd }}$ and $3^{\text {rd }}$ dose and NCA for overall exposure of the 3 doses to accommodate for the multiple-dose regimen (see Methods). The observed and predicted concentration-time profiles used for calculation are shown as Figure 3. The primary endpoint, $\mathrm{AUC}_{0-72 \mathrm{~h} \text {,plasma }} \mathrm{GMR}$, of 0.86 was $14 \%(90 \% \mathrm{Cl}=2-24 \%)$ lower in the $\mathrm{T}+\mathrm{PRO}$ treatment for both TFV and FTC. T +PRO treatment did not achieve equivalent $\mathrm{AUC}_{0-72 h, p l a s m a}$ exposure compared to C IPERGAY HIV PrEP. The PBMC AUC ${ }_{0-72 h}$ GMR of the T +PRO treatment compared to C IPERGAY treatment was $23 \%$ and $29 \%$ lower in the T +PRO treatment for TFV-DP and FTC-TP, respectively (Table 3). Therefore, the overall intracellular exposures of TFV-DP and FTC-TP of the T + PRO treatment were not equivalent to the C IPERGAY treatment.

The single-dose plasma $\mathrm{AUC}_{0-\infty, \mathrm{SD}}$ was also compared to further assess the drug-drug interaction potential of PRO with TDF and FTC (Table 3). The comparison of the $\mathrm{AUC}_{0-\infty, S D}$ showed that the $\mathrm{T}+\mathrm{PRO}$ boosted the overall single-dose exposure by $60-70 \%(\mathrm{TFV} \mathrm{GMR}=1.61,90 \% \mathrm{Cl}=$ 1.47-1.77; FTC GMR $=1.68,90 \% \mathrm{Cl}=1.48-1.89$ ).

This article is protected by copyright. All rights reserved 


\section{Safety}

All healthy volunteers enrolled were included in the safety analysis. The observed AEs are summarized in Table S1. Overall, the reported AEs were considered low grade in severity (grade 1 and 2). Eighteen adverse events occurred during the entire study with 8 of the 15 subjects enrolled experiencing at least one adverse event. Subjects in the T +PRO treatment experienced more adverse events than the C IPERGAY treatment. The most common adverse event was nausea followed by headache. One subject withdrew from the study due to gastroesophageal reflux disease (GERD) which occurred 2 weeks after T +PRO treatment was completed during the washout period and required medical intervention. This incident was determined probable (and not definitely) related to the study intervention since it occurred during the washout period. Of note, the subject reported prior history of gastric reflux.

In the T +PRO treatment, serum creatinine significantly increased from baseline at 24 and 72 hours with mean differences of $0.14 \mathrm{mg} / \mathrm{dL}$ and $0.07 \mathrm{mg} / \mathrm{dL}$, respectively.

This article is protected by copyright. All rights reserved 


\section{Discussion}

This work is the first to report 1) the clinical pharmacokinetic outcomes of TDF/FTC in the presence of PRO and 2) the multiple-dose plasma TFV/FTC and intracellular TFV-DP/FTC-TP disposition profiles for the IPERGAY HIV PrEP regimen.

In the present study, when a single dose of TDF and FTC was given with PRO, PRO increased TFV and FTC plasma exposure to similar extent (by approximately 60-70\%) and decreased total and renal clearances. A decrease in the volume of distribution, V/F, for TFV and FTC was also observed. It should be noted that we have used NCA for estimation of V/F, which inherently limits mechanistic interpretation for orally administered agents. However, inhibition of OAT1/3 by PRO leads to decreased TFV uptake into renal cells available for elimination and thus increases TFV in circulation and may lower V/F because more of the drug is in the blood and less is escaping into renal cells for removal. At 24 hours after dosing, a modest but statistically significant increase in intracellular TFV-DP concentrations $(\sim 30 \% ; p=0.024)$ were noted while FTC-TP concentrations were not different between T +PRO and C IPERGAY treatments. TFV and FTC are predominantly cleared by the kidney via glomerular filtration and active secretion. The glomerular filtration of these drugs is not affected by PRO because the renal clearances exceed the GFR (>90 mL/min) in the presence of PRO. It is well established that OAT-mediated (OAT1 and OAT3) active uptake in to the proximal tubule as well as MRP-mediated (MRP4) efflux to the tubular lumen are responsible for the renal secretion of TFV (8). In vitro studies indicate that PRO inhibits OAT1 and OAT3 at therapeutically relevant concentrations. Indeed, PRO is known to alter the pharmacokinetics of several OAT1/OAT3 substrates. Although PRO-mediated clinical drug interactions with "substrates" of MRP4 have been reported, there is no clear evidence in vitro that PRO is an inhibitor of MRP4 $(14,15)$. We noted that PRO also increased serum creatinine, a known substrate of OAT1 and OAT3. It follows that the mechanism by which PRO increased TFV plasma exposure in the present study could be attributed to inhibition of OAT1/OAT3 by PRO. Of note, the effect of PRO on TFV exposure was relatively small ( $60 \%$ increase) compared to its effects on other substrates of OAT1 and OAT3 such as cefoxitin and furosemide, which were over 2 -fold $(16,17)$. This could be in part due to differences in the contribution of active tubular secretion to the total renal excretion or in differences in systemic metabolism which may be affected by PRO. PRO is known reduce clearance of many drugs via inhibition of OAT1 and OAT3 substrates in various drug classes including antivirals (i.e. oseltamivir, ganciclovir, acyclovir), antibacterials (i.e. nafcillin, ciprofloxacin) and antihistamines (i.e. ranitidine, fexofenadine) or inhibition of glucuronidation (i.e. zidovudine) (18). Therefore, such PK interactions

This article is protected by copyright. All rights reserved 
should be taken in to account when evaluating risk/benefit of PRO addition. The contribution of tubular secretion (20-30\%) to the overall excretion of TFV renal excretion is relatively small. In contrast to TFV, the specific renal transporters involved in FTC secretion have not been unequivocally identified. Based on in vitro data, FTC renal secretion is generally believed to be mediated through OCT transporters on the basolateral membrane $(8,19)$. In this context, the significant increase in FTC exposure by PRO observed in the present study was unexpected. OAT3 was also implicated in the uptake transport of FTC in vitro, although the primary data were not available to evaluate this claim (19). The specific rate-limiting active transporter responsible for FTC renal secretion remains to be established. Although PRO is generally believed to be selective inhibitor of OATs, the possibility that it may also inhibit OCTs cannot be ruled out. Additional studies are needed to confirm the specific mechanisms underlying this observed drug-drug interaction.

Intracellular concentrations of TFV-DP and FTC-TP from single dose $600 \mathrm{mg}$ TDF / $400 \mathrm{mg}$ FTC at $24 \mathrm{~h}$ were similar to published observations (20). After single dose TDF and PRO, TFV-DP concentration remains unchanged over 72 hours demonstrating a long intracellular half-life. After multiple doses, our data shows TFV-DP demonstrates a non-linear accumulation in PBMC. It is reported that TFV-DP has a $t_{1 / 2}$ of 2 days after a single dose and 4-7 days at steady state in PBMC (20-24). Our data suggests that when TFV is given with PRO, TFV-DP $t_{1 / 2}$ is longer than 3 days. In contrast, after single dose FTC and PRO, there is a decline in FTC-TP concentration between $24 \mathrm{~h}$ and $72 \mathrm{~h}$ in a linear and first-order fashion suggesting our sampling times captured the elimination phase of FTC. This would be supported by the literature suggesting FTC-TP $t_{1 / 2}$ is 17-33 hours (23, 24). It is reported that FTC-TP achieves maximum concentrations within 2-4 hours. Thus, PBMC sampling at an earlier time (i.e. 2-4 $\mathrm{h}$ after dose) may better capture maximum FTC-TP concentrations (23). For TFV-DP, the reported median time to achieve $C_{\max }$ is 12 hours (IQR: 8-96h) (23) suggesting we may have caught some, but not all individuals $C_{\max }$ for TFV-DP at the 24 hours sample collection (GM $15 \mathrm{fmol} / 10^{6}$ cells for $C$ treatment; $20 \mathrm{fmol} / 10^{6}$ for $T$ treatment) as well. This is a limitation for analysis if $\mathrm{C}_{\max }$ of active metabolites is a pertinent parameter for antiretroviral activity. FTC-TP intracellular accumulation is unclear after multiple doses, with a mild increase at $48 \mathrm{~h}$ and decrease at 72h. FTC-TP was reported to have a 1.7-fold accumulation in PBMC from single dose to steady state ( $\sim 3$ days) (22). It is difficult to draw conclusions for FTC-TP concentrations in the control arm due to differences in sampling time.

The overall probenecid-boosted TFV and FTC AUC $0-72 \mathrm{~h}$,plasma and $\mathrm{AUC}_{0-72 \mathrm{~h}, \mathrm{PBMC}}$ did not meet the pre-defined equivalence endpoints when compared to the IPERGAY HIV PrEP regimen. Given a 
4 to 12 hours half-life of probenecid (25), it is not surprising that the effects of probenecid on TFV and FTC would be much less evident during the $2^{\text {nd }}$ or $3^{\text {rd }}$ days of the study. An important limitation of our study is that estimation of the true elimination phase in the C IPERGAY treatment was not accurately captured due to the study design. Since nonparametric superposition is based on the accumulation ratio computed from the terminal phase slope, $k_{e l}$, and this is a cross-over study, we used each participant's $k_{e l}$ from the T +PRO treatment for nonparametric superposition of the $2^{\text {nd }}$ and $3^{\text {rd }}$ dose in the C IPERGAY treatment to estimate $A \cup C_{0-72 h \text {,plasma }}$ under the assumption that the $k_{\text {el }}$ for each individual in the T +PRO treatment was not effected by PRO. This is supported by the terminal $\mathrm{t}_{1 / 2}$ from the $\mathrm{T}+\mathrm{PRO}$ treatment corresponding well with literature data (18) for TFV $(\sim 18 \mathrm{~h})$ and FTC $(\sim 10 \mathrm{~h})$ without PRO, suggesting PRO did not impact the overall $t_{1 / 2}$ and provided justification for the method. Therefore, we believe that this estimation is reasonable for the primary endpoint.

The required concentrations of TFV-DP and FTC-TP in PBMCs for On-demand PrEP to prevent HIV infection is not well established, making it difficult to predict the clinical relevance of single-dose PRO-boosted TDF. Anderson et al. established previously TFV-DP concentrations above $16 \mathrm{fmol} / 10^{6}$ cells (95\% Cl: $\left.3-28\right)$ and FTC-TP above 3.7 (95\% Cl: $\left.1.2-6.1\right) \mathrm{pmol} / 10^{6}$ cells in PBMCs were associated with a $90 \%$ reduction in the risk of acquiring HIV-1 infection for daily TDF/FTC HIV PrEP (26). However, these intracellular efficacy "cut-off" concentrations are difficult to confidently apply outside of this study by Anderson et al. due to unknown drug degradation parameters from prolonged storage of their PBMC samples whereas in our study PBMC intracellular concentrations were measured relatively quickly. Rectal mucosal tissue is one of the first sites susceptible for HIV transmission in MSM and TGW. Active drug exposure at initial sites vulnerable for HIV acquisition is crucial for non-daily HIV PrEP (27). Literature suggests the TFV-DP rectal mononuclear cell to intracellular (PBMC) concentrations are more than 10 times greater and reach steady state within 5 days $(23,27)$, while FTC-TP rectal concentrations are negligible but highest in PBMC cells reaching maximum concentrations within 2-4 hours $(20,23)$. Due to financial and practical considerations, rectal biopsies were not obtained in this study but would undoubtedly contribute to interpretation of HIV PrEP pharmacokinetic analysis. This may suggest TDF is the moiety in TDF/FTC that provides prolonged rectal HIV protection while FTC may provide rapid PBMC protection in non-daily HIV PrEP regimens. In this context, the clinical relevance of our findings require further investigation; until then we strongly recommend that the PRO regimen not be employed clinically given the concerns for lack of bioequivalence.

This article is protected by copyright. All rights reserved 
Although the PRO regimen was tolerated, there were more frequent episodes of adverse events, in particular nausea, with the T PRO arm. This may have practical implications as a PrEP agent would have to be extremely well-tolerated to allow sufficient adherence to be clinically effective. Of note, PRO given with zidovudine has previously been shown to lead to adverse systemic cutaneous reactions in HIV-positive patients (28). Thus, if a probenecid based PrEP regimen failed, there would be a risk of similar severe reactions developing in the newly infected person. Thus, a probenecid based regimen would have to be proven well-tolerated in large trials before it could be practically employed.

In summary, we have shown for the first time that PRO reduces the renal secretion of TFV via inhibition of OAT1 and OAT3. We also observed that PRO increased plasma exposure of FTC. This interaction was unexpected and mechanistic studies are warranted. As nondaily HIV PrEP with TDF/FTC becomes more widely accepted, therapy optimized for patient adherence, reduce pill burden, and cost may provide the foundation for HIV PrEP worldwide, particularly in countries with resource- and healthcare- limited settings. Our findings provide proof-of-concept that inhibition of renal transporters alter the disposition of TFV/FTC. However, whether these data would be adequate to recommend PRO-boosted TFV/FTC regimen as simplified and cost-effective PrEP needs further study, particularly to confirm the required intracellular concentrations of these agents for On-demand HIV PrEP including in other target tissues (e.g., rectal).

This article is protected by copyright. All rights reserved 


\section{Study Highlights}

\section{What is the current knowledge on the topic?}

A combination pill containing 300 mg TDF / 200 mg FTC is highly efficacious in preventing new HIV infections when given daily or as multiple-dose 'on demand' drug administration. Tenofovir (TFV), major metabolite of TDF found in circulation, undergoes renal secretion via OAT 1 and 3. Probenecid is a known inhibitor of OAT 1 and 3.

\section{What question did this study address?}

We hypothesize that inhibition of OAT 1 and OAT 3 by probenecid will increase TFV plasma exposure and the intracellular concentrations of its active metabolite (TFV-diphosphate), providing clinical benefit to allow a less expensive, single-dose HIV prevention regimen of TDF/FTC.

\section{What does this study add to our knowledge?}

Probenecid boosted plasma exposure of TFV and initial intracellular exposure of its active metabolite. Probenecid also increased plasma FTC exposure. However, formal bioequivalence was not achieved.

\section{How might this change clinical pharmacology or translational science?}

While the interaction of TFV with probenecid was expected, an unexpected interaction between probenecid and FTC was identified. Additional investigation will need to determine the clinical utility of probenecid-boosted TDF/FTC for HIV prevention.

\section{Author Contributions}

S.N.L., B.T.G., Z.D., J.B.L., P.L.A., R.F.B., and S.K.G. wrote the manuscript; Z.D., B.T.G., and S.K.G. designed the research; S.N.L. and B.T.G. performed the research; S.N.L., B.T.G., J.B.L., and L.R.B. analyzed the data; B.T.G., J.B.L., Z.D., P.L.A., and LRB contributed new reagents/analytical tools. 


\section{References}

(1) Sidebottom, D., Ekstrom, A.M. \& Stromdahl, S. A systematic review of adherence to oral pre-exposure prophylaxis for HIV - how can we improve uptake and adherence? BMC Infect Dis 18, 581 (2018).

(2) Centers for Disease Control and Prevention: US Public Health Service: Preexposure prophylaxis for the prevention of HIV infection in the United States-2017 Update: a clinical practice guideline. <https://www.cdc.gov/hiv/pdf/risk/prep/cdc-hiv-prep-guidelines-2017.pdf (2018). Accessed April 1 2019.

(3) (2018). The impact of pre-exposure prophylaxis with TDF/FTC on HIV diagnoses, 2012-2016, United States. 22nd International AIDS Conference.

(4) Grant, R.M. et al. Preexposure chemoprophylaxis for HIV prevention in men who have sex with men. N Engl J Med 363, 2587-99 (2010).

(5) Molina, J.M. et al. On-Demand Preexposure Prophylaxis in Men at High Risk for HIV-1 Infection. $N$ Engl J Med 373, 2237-46 (2015).

(6) Grant, R.M. et al. Daily and Nondaily Oral Preexposure Prophylaxis in Men and Transgender Women Who Have Sex With Men: The Human Immunodeficiency Virus Prevention Trials Network 067/ADAPT Study. Clin Infect Dis 66, 1712-21 (2018).

(7) Robbins, N., Koch, S.E., Tranter, M. \& Rubinstein, J. The history and future of probenecid. Cardiovasc Toxicol 12, 1-9 (2012).

(8) Anderson, P.L., Kiser, J.J., Gardner, E.M., Rower, J.E., Meditz, A. \& Grant, R.M. Pharmacological considerations for tenofovir and emtricitabine to prevent HIV infection. J Antimicrob Chemother 66, 240-50 (2011).

(9) Cottrell, M.L. et al. A Translational Pharmacology Approach to Predicting Outcomes of Preexposure Prophylaxis Against HIV in Men and Women Using Tenofovir Disoproxil Fumarate With or Without Emtricitabine. J Infect Dis 214, 55-64 (2016).

(10) Delahunty, T., Bushman, L., Robbins, B. \& Fletcher, C.V. The simultaneous assay of tenofovir and emtricitabine in plasma using LC/MS/MS and isotopically labeled internal standards. $J$ Chromatogr $B$ Analyt Technol Biomed Life Sci 877, 1907-14 (2009).

(11) Bushman, L.R. et al. Determination of nucleoside analog mono-, di-, and tri-phosphates in cellular matrix by solid phase extraction and ultra-sensitive LC-MS/MS detection. J Pharm Biomed Anal 56, 390-401 (2011).

This article is protected by copyright. All rights reserved 
(12) Baheti, G., Kiser, J.J., Havens, P.L. \& Fletcher, C.V. Plasma and intracellular population pharmacokinetic analysis of tenofovir in HIV-1-infected patients. Antimicrob Agents Chemother 55, 5294-9 (2011).

(13) Gilead Sciences, I. Genvoya Clinical Pharmacology and Biopharmaceutics Review(s). <https://www.accessdata.fda.gov/drugsatfda_docs/nda/2015/2075610rig1s000ClinPharmR.pdf (2014). Accessed April 12019.

(14) Reid, G. et al. Characterization of the transport of nucleoside analog drugs by the human multidrug resistance proteins MRP4 and MRP5. Mol Pharmacol 63, 1094-103 (2003).

(15) van Aubel, R.A., Smeets, P.H., Peters, J.G., Bindels, R.J. \& Russel, F.G. The MRP4/ABCC4 gene encodes a novel apical organic anion transporter in human kidney proximal tubules: putative efflux pump for urinary cAMP and cGMP. J Am Soc Nephrol 13, 595-603 (2002).

(16) Vlasses, P.H., Holbrook, A.M., Schrogie, J.J., Rogers, J.D., Ferguson, R.K. \& Abrams, W.B. Effect of orally administered probenecid on the pharmacokinetics of cefoxitin. Antimicrob Agents Chemother 17, 847-55 (1980).

(17) Vree, T.B., van den Biggelaar-Martea, M. \& Verwey-van Wissen, C.P. Probenecid inhibits the renal clearance of frusemide and its acyl glucuronide. Br J Clin Pharmacol 39, 692-5 (1995).

(18) Feng, B. et al. Quantitative prediction of renal transporter-mediated clinical drug-drug interactions. Mol Pharm 10, 4207-15 (2013).

(19) Nakatani-Freshwater, T. \& Taft, D.R. Renal excretion of emtricitabine I: effects of organic anion, organic cation, and nucleoside transport inhibitors on emtricitabine excretion. J Pharm Sci 97, 540110 (2008).

(20) Fonsart, J. et al. Single-dose pharmacokinetics and pharmacodynamics of oral tenofovir and emtricitabine in blood, saliva and rectal tissue: a sub-study of the ANRS IPERGAY trial. $J$ Antimicrob Chemother 72, 478-85.

(21) Louissaint, N.A. et al. Single dose pharmacokinetics of oral tenofovir in plasma, peripheral blood mononuclear cells, colonic tissue, and vaginal tissue. AIDS Res Hum Retroviruses 29, 1443-50 (2013).

(22) Seifert, S.M. et al. Dose response for starting and stopping HIV preexposure prophylaxis for men who have sex with men. Clin Infect Dis 60, 804-10.

(23) Seifert, S.M. et al. Intracellular Tenofovir and Emtricitabine Anabolites in Genital, Rectal, and Blood Compartments from First Dose to Steady State. AIDS Res Hum Retroviruses 32, 981-91.

This article is protected by copyright. All rights reserved 
(24) Chen, X. et al. Model Linking Plasma and Intracellular Tenofovir/Emtricitabine with Deoxynucleoside Triphosphates. PloS One 11, e0165505 (2016).

(25) Cunningham, R.F., Israili, Z.H. \& Dayton, P.G. Clinical pharmacokinetics of probenecid. Clin Pharmacokinet 6, 135-51 (1981).

(26) Anderson, P.L. et al. Emtricitabine-tenofovir concentrations and pre-exposure prophylaxis efficacy in men who have sex with men. Sci Transl Med 4, 151ra25 (2012).

(27) Anderson, P.L., Garcia-Lerma, J.G. \& Heneine, W. Nondaily preexposure prophylaxis for HIV prevention. Curr Opin HIV AIDS 11, 94-101.

(28) Petty, B.G., Kornhauser, D.M. \& Lietman, P.S. Zidovudine with probenecid: a warning. Lancet (London, England) 335, 1044-5 (1990).

\section{Figure Legends}

Figure 1. GM and 95\% Cl for plasma concentration-time profiles for TFV (top) and FTC (bottom) concentrations for the T +PRO (open blue squares) and C IPERGAY (closed black circles) treatments.

Figure 2. GM and $95 \% \mathrm{Cl}$ intracellular concentration-time profiles of TFV-DP (top) and FTC-TP (bottom) concentrations for the T +PRO (open blue squares) and C IPERGAY (closed black circles) treatments. 
Figure 3. Individual concentration-time profiles for multiple-doses of TDF and FTC in C IPERGAY treatment with predicted additional doses (grey lines) overlay observed data for TFV (black solid circles) and FTC (blue open squares).

Figure 4. Study Design. Study periods are shown in days 1-4. Study interventions are (1) "T" test +PRO Treatment $=2 \mathrm{~g}$ PRO + $600 \mathrm{mg}$ TDF/ $400 \mathrm{mg}$ FTC at $\mathrm{t}=0 \mathrm{~h}$ or (2) "C" control IPERGAY Treatment $=600 \mathrm{mg}$ TDF $/ 400 \mathrm{mg} \mathrm{FTC}$ at $\mathrm{t}=0 \mathrm{~h} ; 300 \mathrm{mg}$ TDF $/ 200 \mathrm{mg}$ FTC at $\mathrm{t}=24,48 \mathrm{~h}$. Treatment regimens were separated by $\geq 6$ weeks wash-out period.

\section{Tables}

Table 1. Single-dose pharmacokinetics of TFV and FTC in the test (T +PRO) and control (C -PRO) treatments represented as geometric mean (GM) or geometric mean ratio (GMR, T/C) and $90 \%$ confidence intervals $(90 \% \mathrm{Cl})$. * represents median values; a is recovered up to 24 hour data; ${ }^{\text {b }} 72$ is recovered up to 24 hour data.

Table 2. Geometric mean (GM) intracellular TFV-DP and FTC-TP concentrations in the T +PRO and Control IPERGAY treatments and geometric mean ratio (GMR, T/C) and 90\% confidence intervals $(90 \% \mathrm{Cl})$.

Table 3. Geometric least square mean AUC equivalence test for TFV/TFV-DP and FTC/FTC-TP in the test $(\mathrm{T},+\mathrm{PRO})$ compared to control (C, IPERGAY) treatments and GMR (T/C) with $90 \%$ confidence intervals $(90 \% \mathrm{Cl})$. 


\section{Supplemental Material}

Table S1. Adverse events reported in healthy volunteers in the test ( $T,+P R O)$ and control $(C$, IPERGAY) treatments.

\section{SUPPLEMENTARY MATERIALS}

(Supplemental Material.docx)

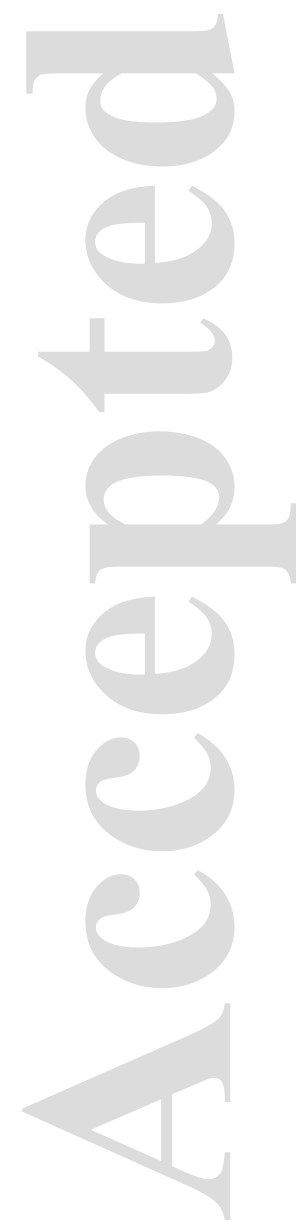

This article is protected by copyright. All rights reserved 
Table 1.

\begin{tabular}{|c|c|c|c|c|c|c|}
\hline \multirow{2}{*}{ PK Parameters } & \multicolumn{3}{|c|}{ Tenofovir (TFV) } & \multicolumn{3}{|c|}{ Emtricitabine (FTC) } \\
\hline & C & $\bar{T}$ & GMR (T/C) & C & $\bar{T}$ & GMR (T/C) \\
\hline \multirow[t]{2}{*}{$C_{\max }(n g / m L)$} & 529 & 538 & 1 & 3426 & 4108 & 1.2 \\
\hline & $(464-602)$ & $(502-577)$ & $(0.91-1.1)$ & $(3137-4806)$ & $(3720-4535)$ & $(1.0-1.4)$ \\
\hline \multirow[t]{2}{*}{$T_{\max }(h)^{*}$} & 1 & 1.5 & & 2 & 2 & \\
\hline & $(1-4)$ & $(1-2)$ & & $(1-4)$ & $(1-4)$ & \\
\hline \multirow[t]{2}{*}{$\mathrm{AUC}_{0-\mathrm{INF}, \mathrm{SD}}\left(\mathrm{ng}^{\star} \mathrm{h} / \mathrm{mL}\right)$} & 3958 & 6444 & 1.6 & 20635 & 34030 & 1.7 \\
\hline & $(3618-4331)$ & $(5827-7127)$ & $(1.5-1.8)$ & $(18984-22431)$ & (30886-37494) & $(1.5-1.9)$ \\
\hline \multirow[t]{2}{*}{$\mathrm{AUC}_{0-24 \mathrm{~h}, \mathrm{SD}}\left(\mathrm{ng}^{\star} \mathrm{h} / \mathrm{mL}\right)$} & 3026 & 4963 & 1.6 & 19488 & 31482 & 1.6 \\
\hline & $(2799-3271)$ & $(4576-5385)$ & $(1.5-1.8)$ & $(17830-21300)$ & $(28856-34346)$ & $(1.4-1.8)$ \\
\hline \multirow[t]{2}{*}{$\mathrm{CL} / \mathrm{F}(\mathrm{L} / \mathrm{h})$} & 69 & 43 & 0.61 & 19 & 12 & 0.61 \\
\hline & $(50-95)$ & $(29-63)$ & $(0.56-0.67)$ & $(18-21)$ & $(11-13)$ & $(0.54-0.69)$ \\
\hline \multirow[t]{2}{*}{$k_{e l}(1 / h)$} & 0.055 & 0.059 & & 0.1 & 0.099 & \\
\hline & $(0.050-0.60)$ & $(0.054-0.064)$ & & $(0.087-0.12)$ & $(0.090-0.11)$ & \\
\hline \multirow[t]{2}{*}{$t_{1 / 2}(h)^{a}$} & 13 & 12 & & 6.9 & 7 & \\
\hline & $(12-14)$ & $(11-13)$ & & $(5.9-7.9)$ & $(6.3-7.7)$ & \\
\hline \multirow[t]{2}{*}{$t_{1 / 2}(h)^{b}$} & & 18 & & & 10 & \\
\hline & & $(16-19)$ & & & $(8-12)$ & \\
\hline \multirow[t]{2}{*}{$V_{z} / F(L)$} & 1278 & 676 & 0.57 & 192 & 119 & 0.62 \\
\hline & $(886-1841)$ & $(495-923)$ & $(0.51-0.64)$ & $(158-231)$ & $(107-132)$ & $(0.53-0.73)$ \\
\hline \multirow[t]{2}{*}{$\%$ dose in urine ${ }^{c}$} & 17 & 17 & & 62 & 68 & \\
\hline & $(14-21)$ & $(15-21)$ & & (53-72) & $(57-80)$ & \\
\hline \multirow[t]{2}{*}{$\mathrm{Cl}_{\text {renal }}{ }^{\mathrm{C}}(\mathrm{mL} / \mathrm{min})$} & 257 & 158 & 0.60 & 210 & 153 & 0.7 \\
\hline & $(212-310)$ & $(131-190)$ & $(0.52-0.73)$ & $(175-252)$ & $(124-190)$ & $(0.54-0.89)$ \\
\hline
\end{tabular}


Table 1. Single-dose pharmacokinetics of TFV and FTC in the test (T +PRO) and control (C -PRO) treatments represented as geometric mean (GM) or geometric mean ratio (GMR, T/C) and 90\% confidence intervals (90\% Cl). Plasma pharmacokinetic parameters are recovered up to 24 hour data; * represents median values; ${ }^{a}$ is recovered up to 24 hour data; ${ }^{b}$ is recovered up to 72 hour data; ${ }^{c}$ urine pharmacokinetic parameters were estimated from 0-24h urine collection.

This article is protected by copyright. All rights reserved 
Table 2.

\begin{tabular}{lcccccc} 
& \multicolumn{3}{c}{ Tenofovir-DP } & \multicolumn{3}{c}{ Emtricitabine-TP } \\
\cline { 2 - 7 } PBMC Concentration & $\mathbf{C}$ & $\mathbf{T}$ & GMR (T/C) & $\mathbf{C}$ & $\mathbf{T}$ & GMR (T/C) \\
\hline $\mathbf{C}_{\mathbf{2 4 h}}$ (fmol/106 cells) & 15 & 20 & 1.3 & 1861 & 2077 & 1.1 \\
& $(13-19)$ & $(17-23)$ & $(1.0-1.6)$ & $(1523-2274)$ & $(1716-2512)$ & $(0.88-1.4)$ \\
$\mathbf{C}_{\mathbf{4 8 h}}$ (fmol/106 cells) & 32 & 23 & 0.7 & 2029 & 1103 & 0.54 \\
& $(26-19)$ & $(18-28)$ & $(0.52-0.94)$ & $(1569-2624)$ & $(922-1320)$ & $(0.40-0.73)$ \\
$\mathbf{C}_{\mathbf{7 2 h}}$ (fmol/106 cells) & 34 & 21 & 0.6 & 1671 & 639 & 0.38
\end{tabular}

Table 2. Geometric mean (GM) intracellular TFV-DP and FTC-TP concentrations in the T +PRO and Control IPERGAY treatments and geometric mean ratio (GMR, T/C) and $90 \%$ confidence intervals $(90 \% \mathrm{Cl})$. 
Table 3.

\begin{tabular}{|c|c|c|c|c|}
\hline PK Parameter & C & $\mathbf{T}$ & GMR & $90 \% \mathrm{Cl}$ \\
\hline $\begin{array}{c}\text { TFV-DP AUC }{ }_{0-72 h, \text { PBMC }} \\
\text { (fmol` } h / 10^{6} \text { cells) }\end{array}$ & 1.60 & 1.24 & 0.774 & $(0.614-0.976)$ \\
\hline $\begin{array}{c}\text { TFV AUC }_{0-72 h, p l a s m a} \\
\left(\mu g^{\star} h / m L\right)\end{array}$ & 8.05 & 6.97 & 0.865 & $(0.764-0.980)$ \\
\hline $\begin{array}{c}\text { TFV AUC }{ }_{0-\infty, S D} \\
\left(\mu g^{\star} h / m L\right)\end{array}$ & 3.91 & 6.30 & 1.61 & $(1.47-1.77)$ \\
\hline $\begin{array}{l}\text { FTC-TP AUC } \\
\text { (fmol* }{ }^{\star} \mathrm{h} / 10^{6} \text { cells) }\end{array}$ & 117 & 83.4 & 0.712 & $(0.561-0.904)$ \\
\hline $\begin{array}{c}\text { FTC AUC }_{0-72 h} \\
\left(\mu g^{\star} h / m L\right)\end{array}$ & 41.1 & 35.5 & 0.864 & $(0.759-0.984)$ \\
\hline $\begin{array}{c}\text { FTC AUC } \\
\left(\mu g^{*} h / \mathrm{h} / \mathrm{mL}\right)\end{array}$ & 19.6 & 32.8 & 1.68 & $(1.48-1.89)$ \\
\hline
\end{tabular}

Table 3. Geometric least square mean AUC equivalence test for TFV (plasma),TFV-DP (PBMC) FTC (plasma), and FTC-TP (PBMC) in the test (,+ +PRO) compared to control (C, IPERGAY) treatments and GMR (T/C) with $90 \%$ confidence intervals $(90 \% \mathrm{CI})$.

This article is protected by copyright. All rights reserved 

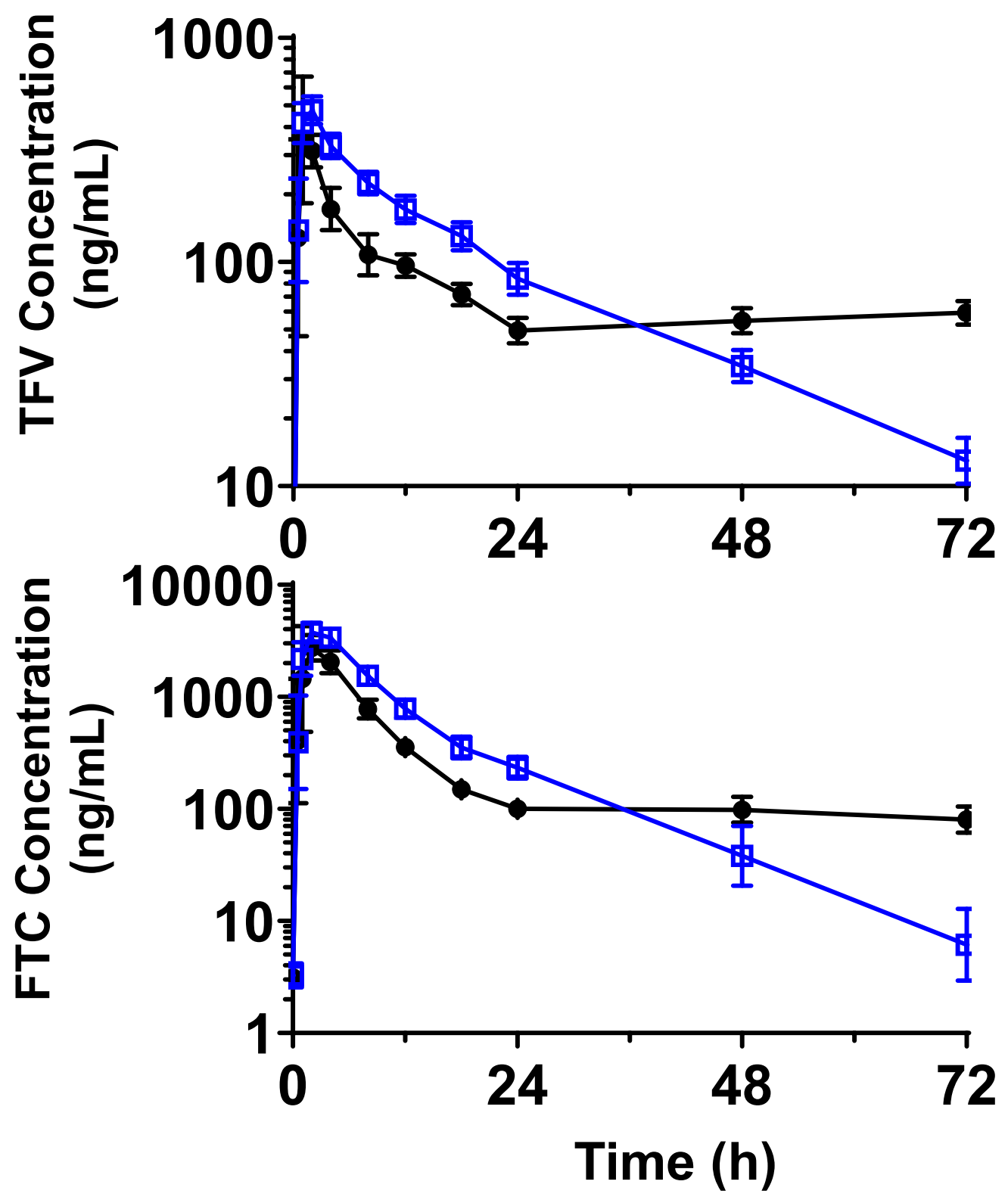

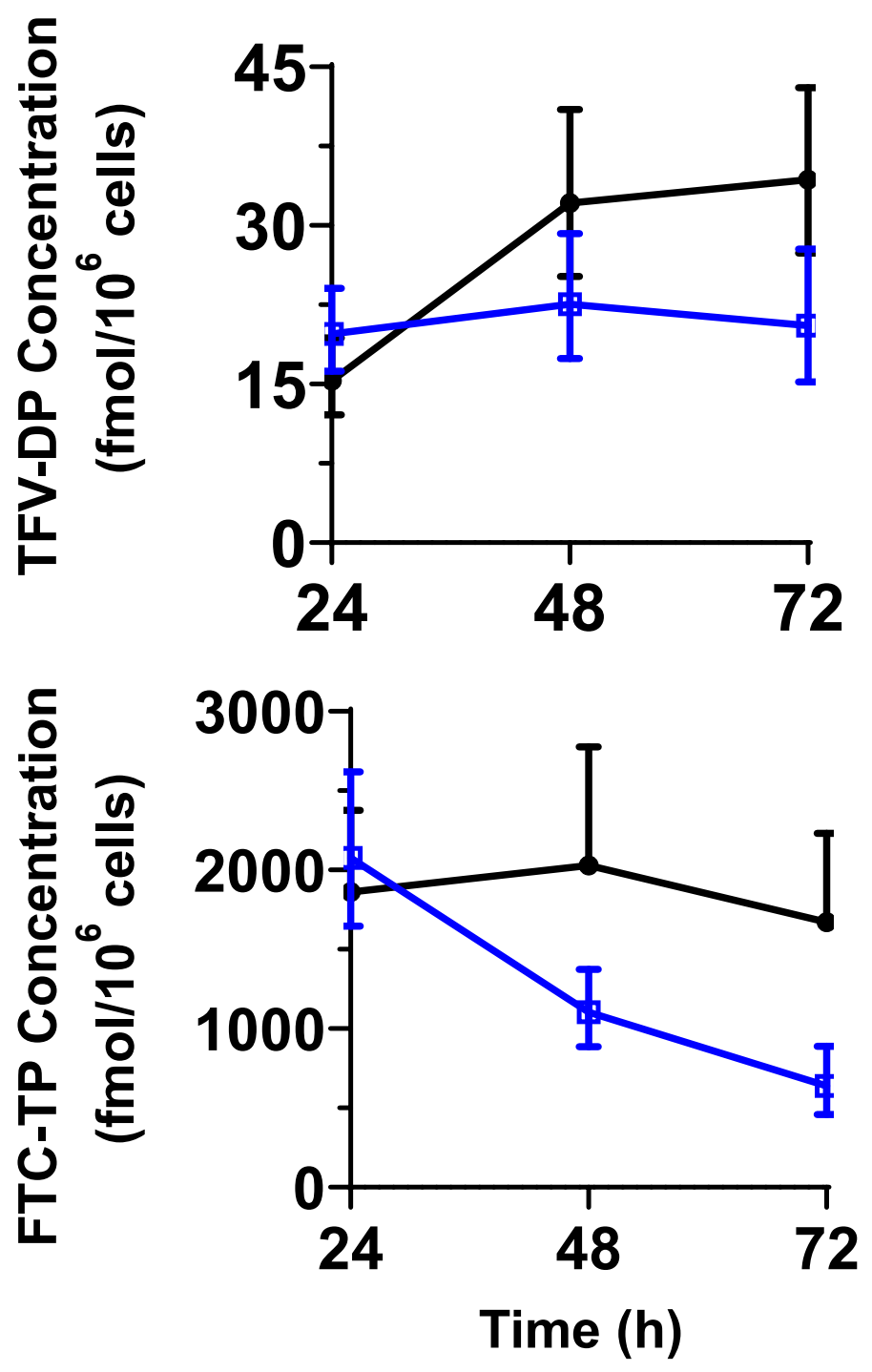

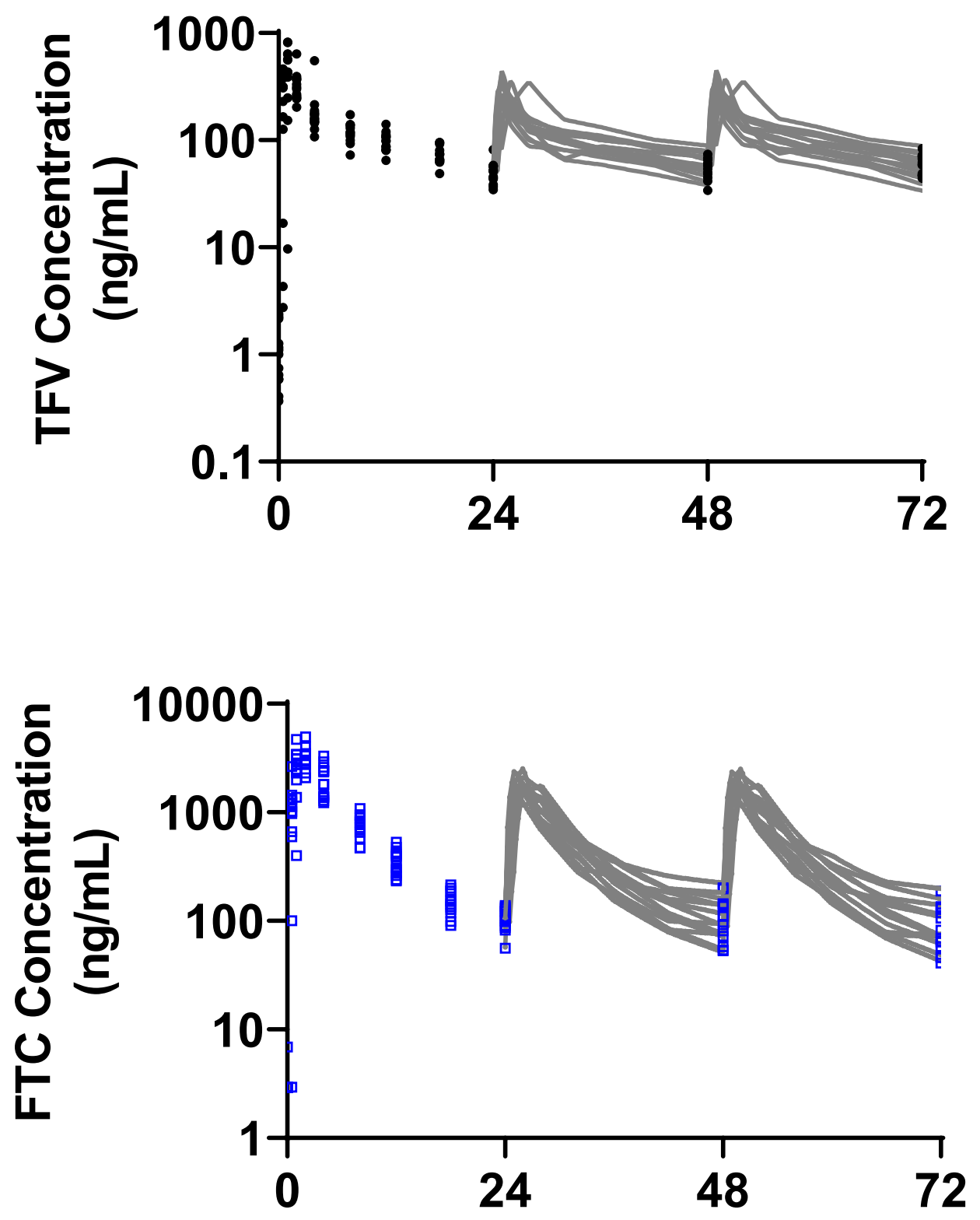


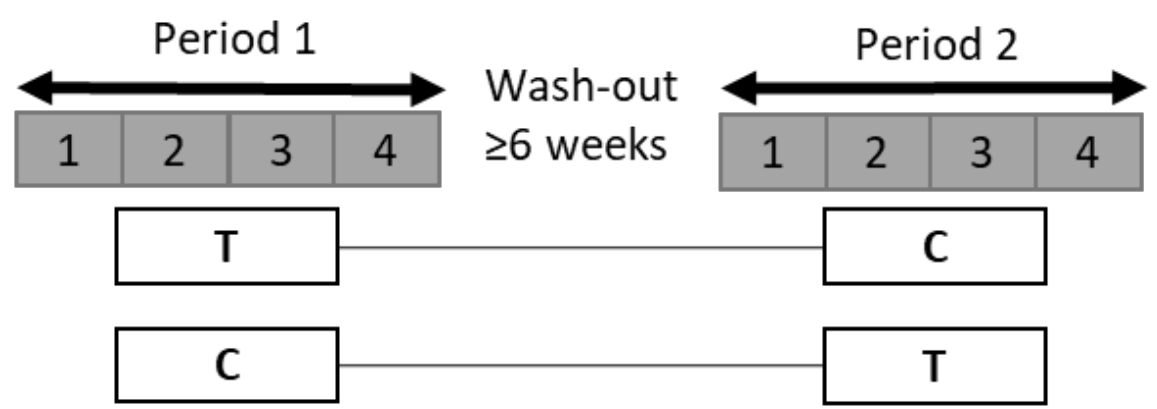

